\title{
Variations in Achilles Tendon Loading With Heel Lift Intervention in Heel-Toe Runners
}

\author{
Sharon J. Dixon \\ University of Exeter
}

\author{
David G. Kerwin \\ University of Bath
}

This study investigated the influence of heel lift interventions on the loading of the Achilles tendon for heel-toe runners. It was hypothesized that the peak Achilles tendon force and peak rate of loading would be reduced by the increase in heel lift, and that the peak Achilles tendon force would occur significantly later in stance. Achilles tendon forces were determined by calculating sagittal-plane ankle joint moments using inverse-dynamics techniques and dividing these moments by Achilles tendon moment arm lengths. Methods for estimating Achilles tendon moment arm length using skin markers were justified via MRI data for one participant. Seven participants underwent running trials under three heel lift conditions: zero, 7.5-mm, and 15-mm heel lift. Average magnitude and occurrence time of peak Achilles tendon force and peak rate of loading were determined for each condition over the 7 participants. Despite group reductions in peak Achilles tendon force and peak rate of loading for the increased heel lift conditions, statistical analysis (ANOVA) revealed no significant differences for these variables, $p>0.05$. Individual participant observations highlighted varied responses to heel lift; both increases and decreases in peak Achilles tendon force were observed. For the group data, the time of peak impact force occurred significantly later in the 15-mm heel lift condition than in the zero heel lift, $p<0.05$. It is suggested that the success of increased heel lift in treating Achilles tendon injury may be due to a later occurrence of peak Achilles tendon force in response to this intervention, reducing Achilles tendon average rate of loading. In addition, the individuality of Achilles tendon peak force changes with heel lift intervention highlights the need for individual participant analysis.

Key Words: magnetic resonance imaging (MRI), heel lift devices, loading rate

\section{Introduction}

The high rate of Achilles tendon injury among distance runners may be attributed to repetitive loading of the tendon (Archambault, Preston Wiley, \& Bray, 1995).

S.J. Dixon is with the School of Exercise and Sport Sciences, University of Exeter, Heavitree Rd, Exeter, EX1 2LU, U.K.; D.G. Kerwin is with the Sports Biomechanics Lab, Dept. of Sport \& Exercise Science, University of Bath, Bath, BA2 7AY, U.K. 
However, it is not known what aspects of the tendon loading are most important; the variables commonly described include peak force, strain, and stress (Clement, Taunton, \& Smart, 1984). In addition to peak loads being a factor in injuries, there is evidence that rates of change in impact force are influenced by shoe manipulation. This has led to the suggestion that the rate of loading of body structures may be relevant when addressing the causes of injury (Lees \& McCullagh, 1984). This suggestion has been supported by Radin, Yang, Riegger, Kish, and O'Conner (1991), who found that persons with anterior knee pain demonstrate higher rates of loading of impact force. A method for investigating the variables most closely linked with injury is to introduce an intervention known to influence the incidence of injury and to study the effect of this manipulation on internal loading. In the present study, Achilles tendon peak force and rate of loading were estimated during running under different heel height conditions.

Shoe inserts designed to raise the heel (i.e., heel lifts) have been recommended for treating Achilles tendon injury (MacLellan, 1984). It is generally assumed that raising the heel relative to the forefoot will help reduce the peak force experienced by the tendon (Clement et al., 1984; Leach, James, \& Wasilewski, 1981). Yet there is no scientific evidence to support this assumption. An earlier study found that three runners with distinct running styles (a heel-toe runner, a midfoot striker, and a forefoot striker) responded differently to heel lift interventions (Dixon \& Kerwin, 1998). One interpretation of this is that runners with different styles respond differently to heel lift manipulation. However, the question of whether runners using a specific running style respond similarly to heel lift manipulation calls for a greater number of participants in a study.

The purpose of the present study was to investigate the influence of increased heel lift on Achilles tendon loading in heel-toe runners. Seven experienced and well-trained heel-toe runners served as participants. Based on suggestions in the literature, it was hypothesized that an increase in heel lift would result in a decrease in Achilles tendon peak force and peak loading rate, and that peak Achilles tendon force would occur later in the stance.

\section{Methods}

Seven female runners volunteered to participate in the study. Magnetic resonance imaging (MRI) data were collected for one participant to provide anatomical data on the location of the Achilles tendon line of action. Biomechanical data were
collected for all seven. Written, informed pant prior to data collection.

The MRI data revealed the orientation of the Achilles tendon relative to the skin. The runner providing the MRI data adopted a supine position with the right foot supported within a padded enclosure. Full range of ankle joint dorsi- and plantar-flexion movement was possible, with support provided beneath the heel and on the medial and lateral sides of the ankle joint. The runner was instructed to move the ankle from a position of maximum ankle dorsiflexion to maximum plantar-flexion, in 6 distinct steps of approximately $10^{\circ}$ (Figure 1). She was required to
hold each ankle angle for approximately 1 minute to allow for the collecting of required images. Approximately 10 seconds elapsed between the collection of data
for each ankle joint orientation. 


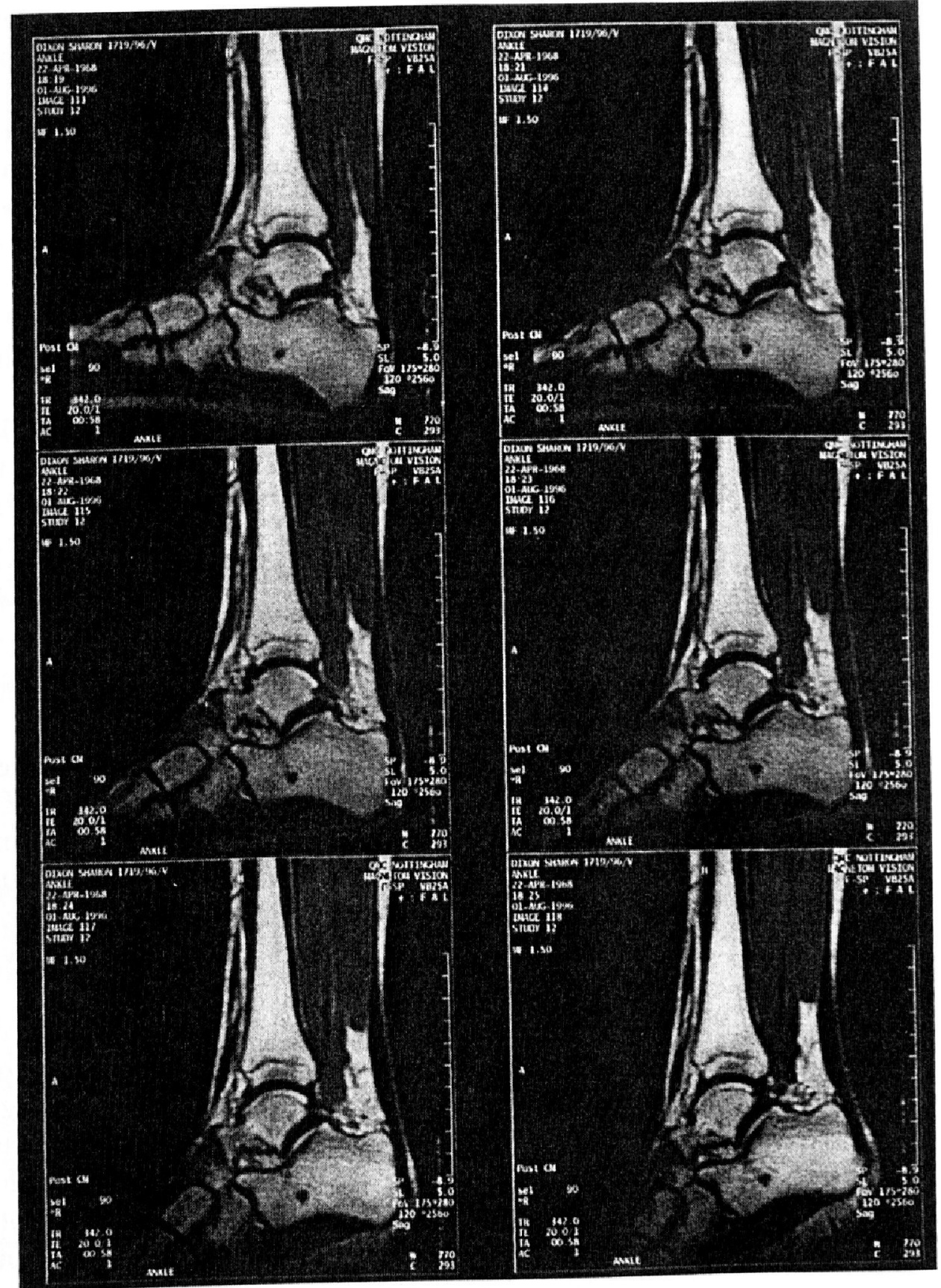

Figure 1 - Sagittal-plane MRI images through the midline of the ankle joint complex for the 6 ankle joint angles. Top left shows peak ankle dorsiflexion, with increasing plantar-flexion as read from left to right.

Sagittal-plane images were used to calculate the distance from the surface of the skin covering the Achilles tendon (posterior aspect) to the Achilles tendon line of action over the range of ankle angles. As shown in Figure 1, the Achilles tendon and the skin covering it have approximately uniform thickness in the sagittal plane for the length of the tendon, between approximately $2 \mathrm{~cm}$ and $15 \mathrm{~cm}$ proximal to tendon insertion. For this length of the tendon, the average distance from the skin surface to the tendon line of action was $3.9 \mathrm{~mm}$. Negligible change was detected in 
this distance throughout the range of ankle joint motion. Markers placed on the skin covering the posterior Achilles tendon could therefore be assumed to provide a line parallel to the Achilles tendon line of action.

The 7 participants for the running trials were well-trained female middledistance runners with a mean mass of $55.6 \mathrm{~kg}(S D 3.5 \mathrm{~kg})$. Preliminary data collection indicated that each one adopted a heel-toe running style when running barefoot at the test speed of $3.83 \mathrm{~m} \cdot \mathrm{s}^{-1}$. The women underwent 10 running trials under each of three heel lift conditions: zero, $7.5-\mathrm{mm}$, and $15-\mathrm{mm}$ heel lift. The order of conditions was randomized across runners, and a minimum of 5 trials was performed for each condition before data collection so as to allow familiarization with the
running condition.

Heel lifts were constructed from a high density EVA material (Frank Lord Ltd., Bolton, UK) and attached to the plantar surface of both feet with light micropore surgical tape. For the zero heel lift condition, two lifts of uniform thickness $7.5 \mathrm{~mm}$ were attached to the rearfoot and the forefoot. This condition raised lift condition was used to control for theel relative to the forefoot. The zero heel baseline condition from control for the effects of lift attachment; it served as a the increased heel lift which to compare the increased heel lift conditions. For thickness $7.5 \mathrm{~mm}$ and $15 \mathrm{~mm}$ were tapered wedges $88 \mathrm{~mm}$ long with maximum

All running and $15 \mathrm{~mm}$ were introduced at the rearfoot.

long, with right trials were conducted along a runway approximately 15 meters with the runway. foot contact with a force plate (Kistler 9281B12) situated flush photocells positioned on either speed was calculated over a 3-m distance using data collection, trials were accepted if the running speed attained was within $+5 \%$ of that specified, and if right foot contact with the force attained was within $\pm 5 \%$ out obvious alterations to running stride.

Force plate data were collected at collected at 120 at were data collection was (MacReflex, Qualisys AB, Göteborg, Sweden). Force plate runner's approach to thered as the first photocell beam was broken during the view of one the force plate. In addition, an infrared LED in the field of synchronized. camera was triggered, allowing the force and kinematic data to be $120-\mathrm{Hz}$ data. Prior to intere obtained by interpolation of the using a cubic spline (Reinsch, 1967). $120-\mathrm{Hz}$ kinematic data were smoothed

were determined using 9-point finite difference methods (Lanczos, 1957).
Reflective markers phalageal joint markers were placed to represent the knee, ankle, and metatarsalon the rear of centers in the sagittal plane. Two additional markers were placed action. The sagittal plane locatide a line parallel to the Achilles tendon line of cal and anterior/posterior coordinates from the was obtained by extracting the vertiprojection error on the 2-D data.

The 2-D sagittal-plane ankle joint moments were calculated using inversedynamics techniques (Winter, 1980). The mass, location of segment mass centers, and segmental moments of inertia about transverse axes through segment mass (1985), in which individusing the regression equations of Zatsiorsky and Seluyanov contribution of the triceps participant height and weight were substituted. The 


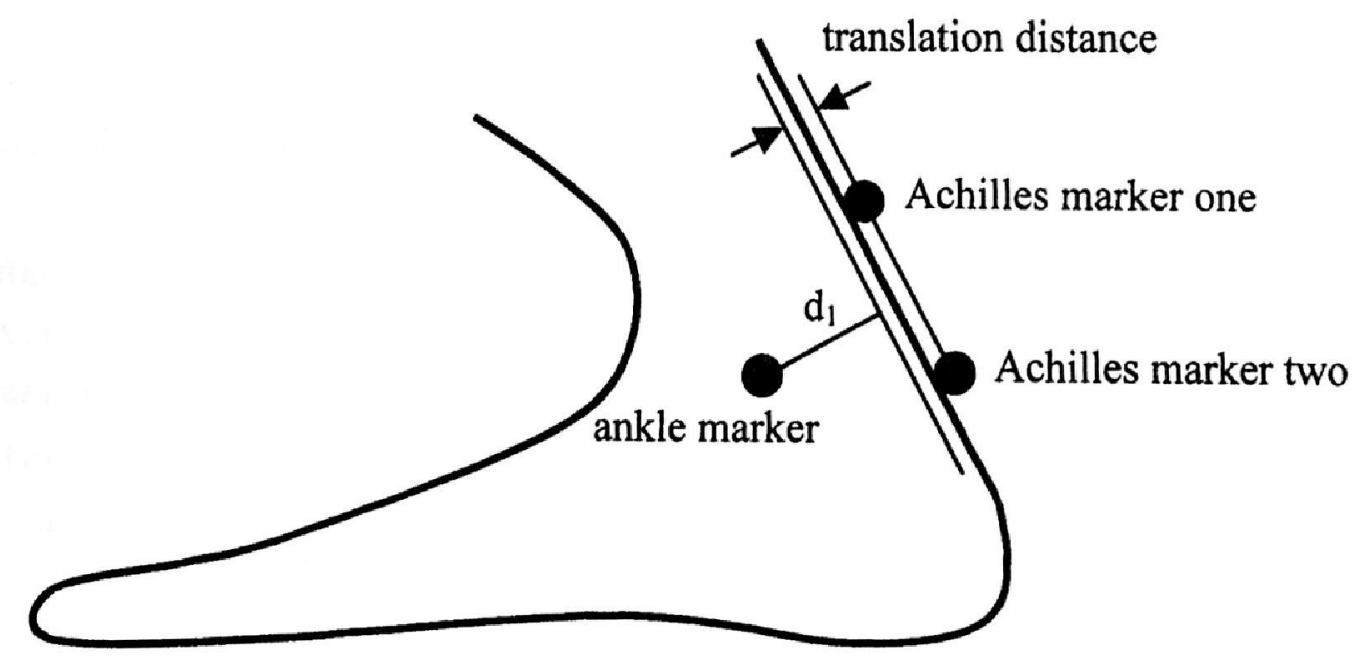

Figure 2 - Location of skin markers for estimating Achilles tendon moment arm $\left(d_{1}\right)$, showing the translation distance for the runner who provided MRI data. This translation distance was scaled for the other runners using relative tendon thickness measurements.

moment was assumed to be $85 \%$, as determined by Scott and Winter (1990) using relative physiological cross-sectional areas.

For each participant, caliper measurements of the Achilles tendon thickness were obtained in the frontal plane at a height of $10 \mathrm{~cm}$ proximal to the foot plantar surface. For the participant providing the MRI data, the tendon's line of action was estimated as being parallel to a line through the center of the tendon markers, translated anteriorly by a distance determined by the radius of the Achilles tendon markers $(5 \mathrm{~mm})$ and the average distance of the skin-to-tendon line of action (3.9 $\mathrm{mm}$ ), as illustrated in Figure 2. The distance of the skin-to-tendon line of action was scaled for other participants using relative caliper measurements of tendon thickness.

Achilles tendon forces were determined for each trial throughout the stance phase of running by dividing the ankle moment by the length of the Achilles tendon moment arm, as described by Dixon and Kerwin (1998). The rate of change of Achilles tendon force (Achilles tendon loading rate) was determined throughout the stance phase using the $1000-\mathrm{Hz}$ Achilles tendon force data. The instantaneous Achilles tendon loading-rate data were smoothed by considering the average rate over 0.01 -s time periods, providing $100-\mathrm{Hz}$ smoothed data. In addition, the average rate of loading of Achilles tendon force was calculated by dividing the magnitude of peak force by the time of its occurrence (from initial ground contact).

Each runner's mean values for magnitude and time of occurrence of peak Achilles tendon force, average Achilles tendon loading rate, and peak Achilles tendon loading rate were calculated for each heel lift condition. Group mean values were calculated and compared across heel lifts using an ANOVA with repeated measures, followed by a post hoc Tukey test, $p<0.05$. Individual results were assessed to identify trends and differences in each runner's response to heel lift manipulation. 
Table 1 Mean (+SD) Values for Group Data

\begin{tabular}{llllll} 
Condition & Zero heel lift & 7.5-mm heel lift & 15-mm heel lift \\
\hline Achilles peak force $(\mathrm{BW})$ & $11.4 \quad(3.7)$ & $10.9(3.4)$ & $10.8 \quad(3.0)$ \\
Time of force $(\mathrm{ms})$ & $101.1 \quad(10.6)$ & $104.6(8.8)$ & $106.1 \quad(12.0)^{*}$ \\
Average loading rate $\left(\mathrm{BW} \cdot \mathrm{s}^{-1}\right)$ & $114.4(46.8)$ & $105.1 \quad(37.4)$ & $103.6(34.8)^{*}$ \\
Peak loading rate $\left(\mathrm{BW} \cdot \mathrm{s}^{-1}\right)$ & $250.4 \quad(121.7)$ & $225.3 \quad(86.8)$ & $241.3 \quad(91.0)$
\end{tabular}

*Significant difference from the zero heel lift condition, $p<0.05$.

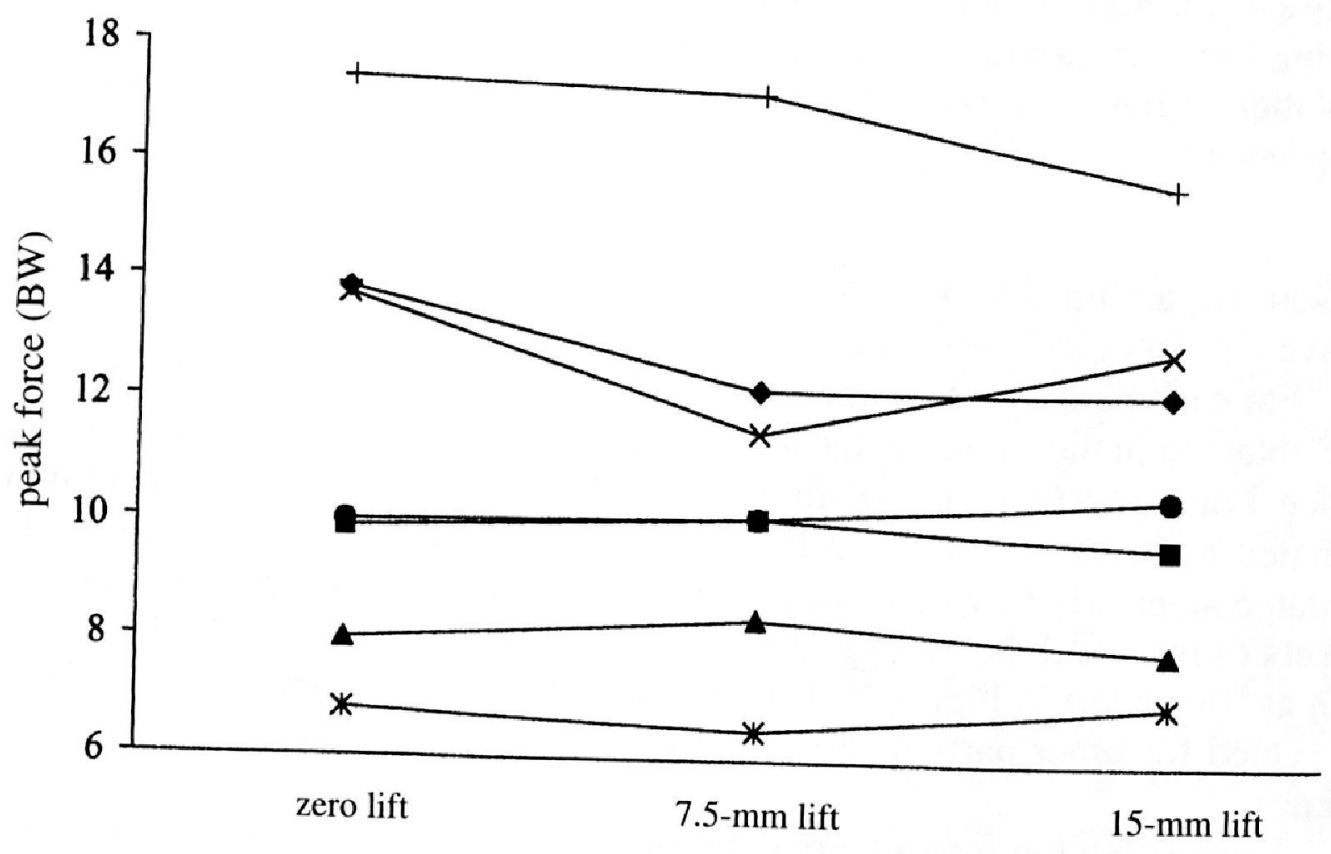

Figure 3 - Peak Achilles tendon force values for individual runners for each heel lift condition. Key: $\uparrow$ Participant 1; $\square$ Participant 2; $\Delta$ Participant 3; $\times$ Participant 4; * Participant 5; Participant 6; + Participant 7 .

\section{Results}

The group data indicated a reduction in peak Achilles tendon force with increased heel lift, although no significant differences were identified (Table 1). Individual participant analysis revealed a varying peak Achilles tendon force response across runners (Figure 3 ). Three runners showed a reduction in peak Achilles tendon force for both of the increased heel lift conditions compared with zero heel lift (Participants $1,4,7)$. Three others demonstrated a small increase for one of the increased heel lift conditions and a small decrease for the other (Participants 2, 3, 5). And 


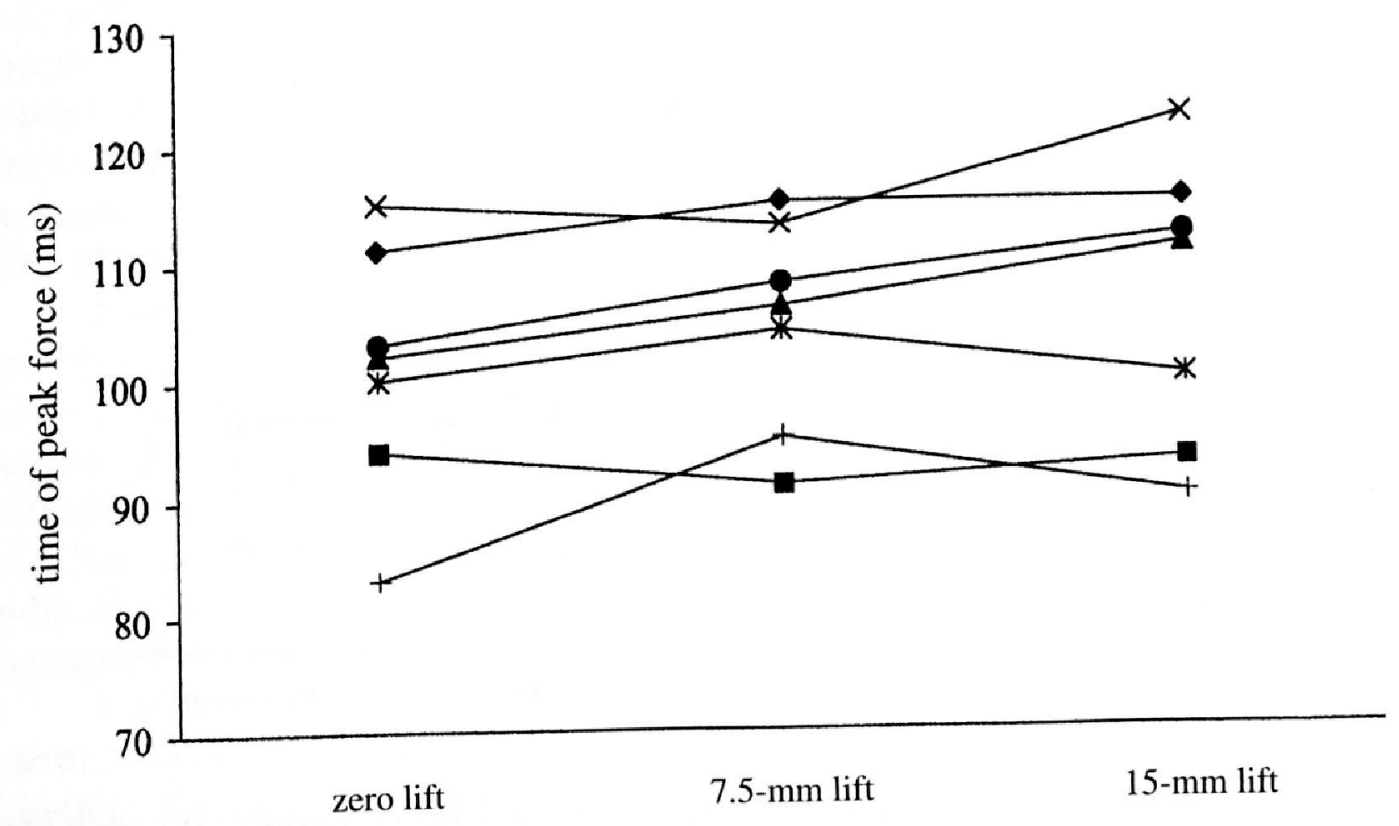

Figure 4 - Time of occurrence of peak Achilles tendon force for individual runners for each heel lift condition. Key: $\bullet$ Participant 1; $\square$ Participant 2; $\Delta$ Participant 3; $\times$ Participant 4; $*$ Participant 5; P Participant 6; + Participant 7.

Participant 6 had an increase in peak Achilles tendon force for both increased heel lift conditions.

For the group, it was found that the time of occurrence of peak force increased with increased heel lift (Table 1). The difference for 15-mm heel lift compared to zero heel lift was significant, $p<0.05$. Consideration of individual results indicated that four runners (Participants 1, 3, 6, 7) showed an increase in the time of occurrence of peak Achilles tendon force for both increased heel lift conditions compared with zero heel lift (Figure 4). Two others demonstrated an increased time of occurrence for one increased heel lift condition but a decrease for the other (Participants 4, 5). Finally, Participant 2 showed a reduced time of occurrence for both increased heel lift conditions. For the group as a whole, the average loading rate was reduced for increased heel lift, with the $15-\mathrm{mm}$ heel lift resulting in a significant reduction compared to the zero heel lift, $p<0.05$. Data on individual runners (Figure 5) indicated that all but Participant 6 showed a reduction in the average rate of loading for the $15-\mathrm{mm}$ heel lift condition compared with zero heel lift, while Participants 1, 3, and 4 failed to show a reduction in this variable for the 7.5-mm heel lift.

The group comparison revealed a reduction in peak loading rate for the increased heel lift conditions compared to zero heel lift (Table 1), but these reductions were not significant, $p>0.05$. The comparison of heel lift conditions for individual runners indicated a varied response in peak loading rate, with no clear trends apparent (Figure 6). Three runners showed a reduction in peak loading rate for both increased heel lift conditions (Participants 2, 5, 7), two others showed an increase for both increased heel lifts (Participants 3 and 4), while Participants 1 and 6 showed a varied response depending on the heel lift condition. 


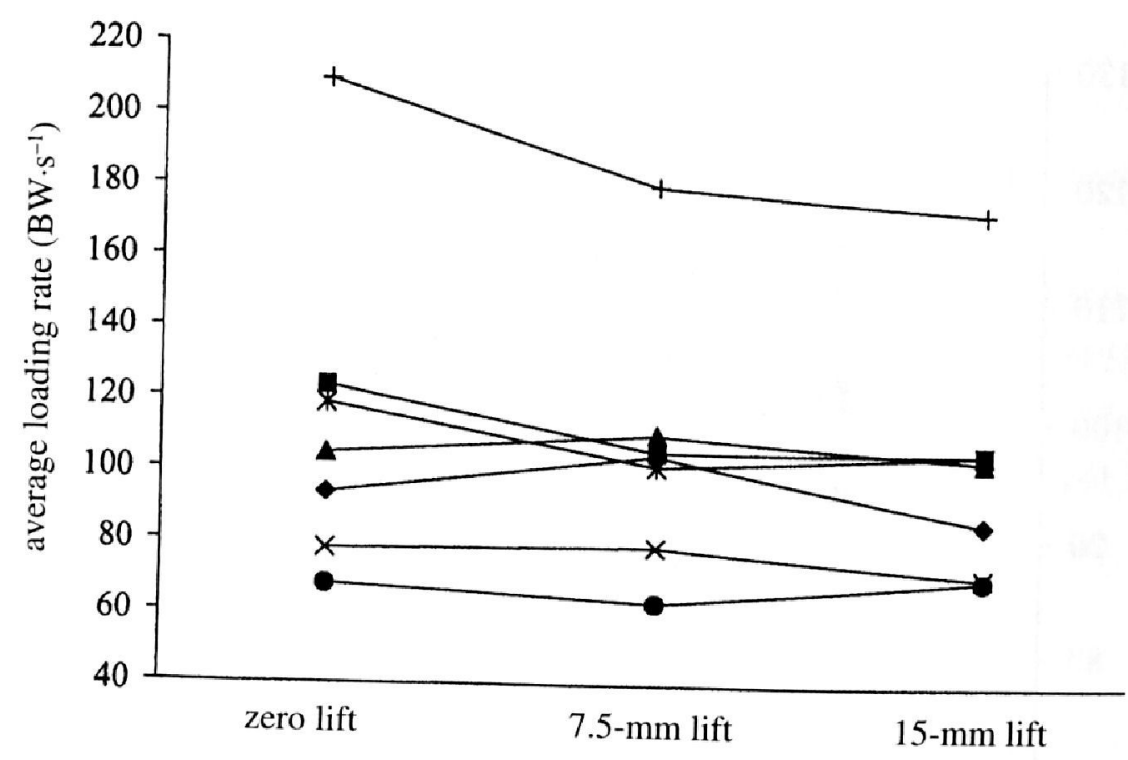

Figure 5 - Average Achilles tendon loading rate for individual runners for each heel lift condition. Key: $\uparrow$ Participant 1; $\square$ Participant 2; $\Delta$ Participant 3; $\times$ Participant 4; $*$ Participant 5; O Participant 6; + Participant 7.

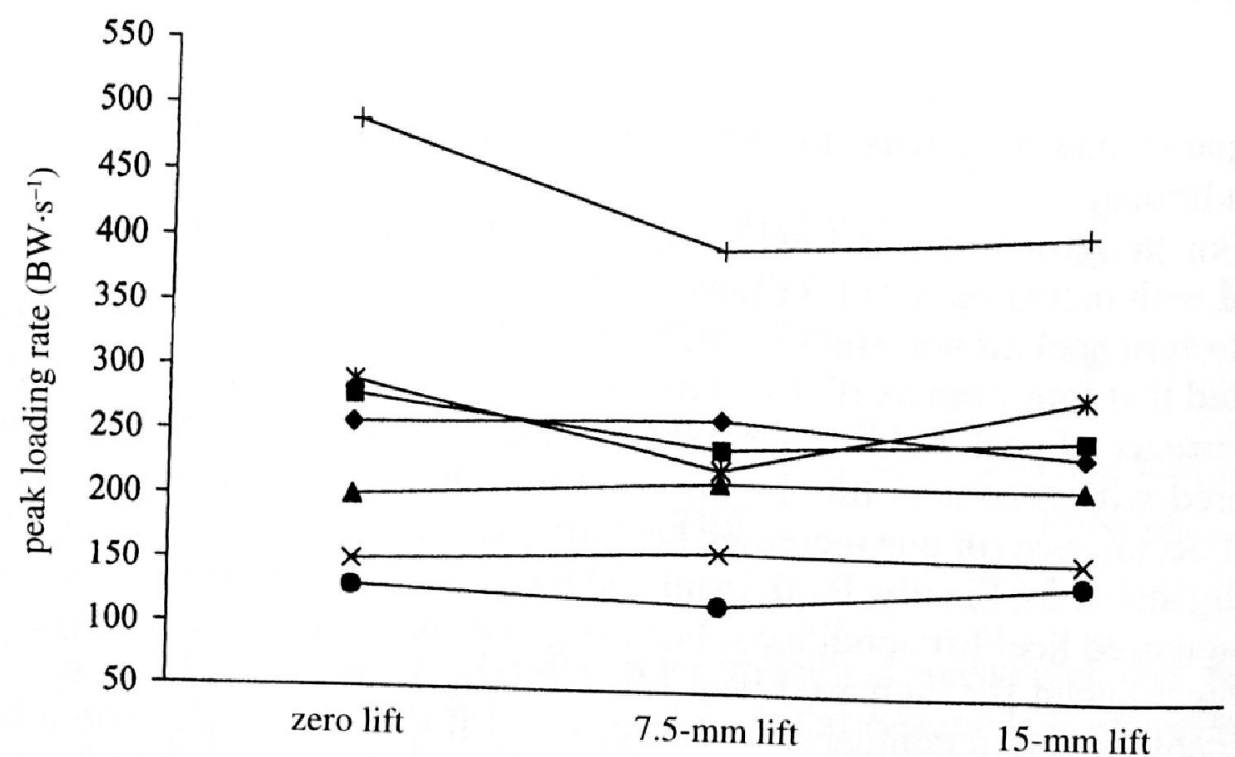

Figure 6 - Peak Achilles tendon loading rate for individual runners for each heel lift condition. Key: $\bullet$ Participant 1; $\square$ Participant 2; $\Delta$ Participant 3; $\times$ Participant 4;
* Participant 5; - Participant 6; + Participant 7 .

\section{Discussion}

The hypothesis that heel-toe runners have a decrease in peak Achilles tendon force with increased heel lift has not been supported by the statistical analysis of group data. However, a tendency for peak force to be reduced is indicated by the mean group values. In addition, individual observations indicate that the majority of run- 

ners demonstrated a reduction in peak force for at least one heel lift condition. It is
therefore suggested that a larger number of participants may have resulted in signifi-
cant group reductions in peak force. However, the increases in peak Achilles tendon cant group reductions in peak force. However, the increases in peak Achilles tendon force observed for some runners when heel lift was introduced indicate that it cannot heel lift intervention, highlighting the requirement for single participant analyses.

A large range of peak Achilles tendon force values was found across runA large range of peak All but Participant 7, demonstrating a force of approximately twice the
value of that of the other six runners, the magnitudes of force are comparable to those in the literature (Burdett, 1982; Komi, 1990). Interestingly, the three runners with the highest peak Achilles tendon force values for zero heel lift were those who had clear reductions in peak force for both heel lift interventions. This may indicate that runners with high Achilles tendon force values are those most likely to respond well to heel lift intervention, in terms of reducing peak force.

In support of the hypothesis that increased heel lift will result in a later occurrence of peak Achilles tendon force, it has been found that the $15-\mathrm{mm}$ heel lift increases the time of occurrence compared to zero heel lift. The absence of a significant effect for the 7.5-mm lift, together with the finding that three runners did not show a reduction in average loading rate for this smaller heel lift, suggests that the $15-\mathrm{mm}$ heel lift may be preferred. If it is assumed that the average rate of tendon loading is associated with injury, this result may be of interest to practitioners in that it suggests a $15-\mathrm{mm}$ heel lift is needed to consistently reduce this variable.

The hypothesis that the peak loading rate of Achilles tendon force would be reduced by increasing the heel lift was not supported by the results of the present study. The lack of a significant group effect and the varied response across individual runners suggest that the mechanism by which a heel lift intervention has been successful in the treatment of Achilles tendon injury is not by a reduction in the magnitude of peak loading rate.

The use of a single-participant approach in addition to the group analysis has highlighted varied responses across runners in some variables, and trends in others. This calls for the use of a multiple single-subject approach in future studies, since the behavior of individual participants would have been overlooked in the present study if only a group analysis had been performed. The varied response Achilles tendon injury, and that individual assessment of athletes is required when prescribing heel lift devices.

The differing responses to heel lift intervention may indicate which runners would show a reduction in injury with this intervention. Not all athletes have been found to respond favorably to the use of an increased heel lift for treating Achilles tendon injury. Perhaps the runners who show a reduced peak Achilles tendon force with increased heel lift are the same ones who would respond favorably if injured. In contrast, the runner who showed a clear increase in Achilles tendon loading with increased heel lift may not demonstrate a reduction in pain with increased heel lift, if injured. The participants in the present study were free from injury at the time of data collection. To support the suggestion that the success of heel lift interventions is participant-specific, the study of athletes with Achilles tendon injury and the monitoring of clinical responses to heel lift intervention is needed along with biomechanical studies. This assumes that Achilles tendon force is the variable of significance when considering the occurrence of Achilles tendon injury. 
It may be that the magnitude of peak Achilles tendon force is not the most important variable when considering the etiology of Achilles tendon injury/The increase in the time to peak Achilles tendon force observed in the present study for increased heel lift conditions suggests that the average rate of tendon loading may be of significance, The majority of runners showed a later occurrence of peak Achilles tendon force with heel lift, and a subsequent reduction in the average rate of loading. During the first half of the stance phase of heel-toe running, the Achilles tendon undergoes eccentric loading due to ankle dorsiflexion and knee flexion during the cushioning phase of the running step. The occurrence of peak Achilles tendon force corresponds with the time of maximum elongation of the tendon. A later occurrence of peak Achilles tendon force therefore indicates an increase in the time period over which the eccentric loading occurs. Since Achilles tendon pain is typically reduced with increased heel lift, the results of this study suggest that the reduction in injury may be the result of a lower average rate of eccentric loading of the tendon.

The use of MRI data indicated that markers can be placed on the skin to represent the orientation of the Achilles tendon, allowing its movement to be monitored with skin markers throughout ground contact in running. The skin markers have been shown to provide a line of action parallel to the Achilles tendon line of action. Appropriate translation and scaling have provided Achilles tendon moment arm lengths throughout the ground contact phase of running. The magnitudes of moment arm are in agreement with the findings of Bobbert, Huijing, and van Ingen Schenau (1986) and of Rugg, Gregor, Mandelbaum, and Chius (1990). The development of reliable, noninvasive methods for estimating Achilles tendon loading in running allows for detailed study of Achilles tendon loading for an unlimited num-

Future technological advances may help increase the accuracy of the methods used in the present study. Differences in the relative movement of the Achilles tendon and the skin markers due to the loads acting on the structures of the foot and ankle during ground contact have been assumed to be small, owing to the small amount of soft tissue between the tendon and markers. The MRI data were collected while that participant was supine, with no loads applied to the plantar surface of the foot. With further development of MRI techniques for collecting data at suitable sampling rates, it may be possible to monitor the movement of internal structures during running. Although the influence of external loads on internal movement was not quantified in the present study, the errors associated with soft tissue vibration are of most concern during the initial heel impact phase when accelerations are high. The Achilles tendon undergoes negligible load during this time; the peak force occurs in the middle of the stance phase when the accelerations are small, thus supporting the skin marker techniques used in the
present study.

The present study has described the investigation of the influence of controlled changes in heel lift. It has clearly demonstrated that heel lift manipulation can influence the loading of the Achilles tendon. It is suggested that future work employ these same biomechanical procedures along with a prospective study to monitor injury and loading response to different heel lift interventions over a certain number of months. It would also be beneficial to have participant-specific MRI data for calculating Achilles tendon moment arms. Finally, the methods de- 
scribed herein are suitable for use in the study of other footwear variations, such as changes in shoe cushioning or frontal plane geometry.

\section{References}

Archambault, J.M., Preston Wiley, J., \& Bray, R.C. (1995). Exercise loading of tendons and overuse injuries. Sports Medicine, 20, 77-89.

Bobbert, M.F., Huijing, P.A., \& van Ingen Schenau, G.J. (1986). A model of the human triceps surae muscle-tendon complex applied to jumping. Journal of Biomechanics, 19, 887-898.

Burdett, R.G. (1982). Forces predicted at the ankle during running. Medicine and Science in Sports and Exercise, 14, 308-316.

Clement, D.B., Taunton, J.E., \& Smart, G.W. (1984). Achilles tendinitis and peritendinitis: Etiology and treatment. American Journal of Sports Medicine, 12, 179-184.

Dixon, S.J., \& Kerwin, D.G. (1998). The influence of heel lift manipulation on Achilles tendon loading in running. Journal of Applied Biomechanics, 14, 374-389.

Komi, P.V. (1990). Relevance of in-vivo force measurements to human biomechanics. Journal of Biomechanics, 23, 23-34.

Lanczos, C. (1957). Applied analysis. New York: Pitman.

Leach, R.E., James, S., \& Wasilewski, S. (1981). Achilles tendinitis. American Journal of Sports Medicine, 9, 93-98.

Lees, A., \& McCullagh, P.J. (1984). A preliminary investigation into the shock absorbency of running shoes and shoe inserts. Journal of Human Movement Studies, 10, 95-106.

MacLellan, G.E. (1984). Skeletal strike transients, measurement, implications and modification by footwear. In E.C. Frederick (Ed.), Sport shoes and playing surfaces (pp. 76-86). Champaign, IL: Human Kinetics.

Radin, E.L., Yang, K.H., Riegger, C., Kish, V.L., \& O'Conner, J.J. (1991). Relationship between lower limb dynamics and knee joint pain. Journal of Orthopaedic Research, 9, 398-405.

Reinsch, C.H. (1967). Smoothing by spline functions. Numerische Mathematik, 10, 177 183.

Rugg, S.G., Gregor, R.J., Mandelbaum, B.R, \& Chius, L. (1990). In vivo moment arm measurement calculations at the ankle joint using magnetic resonance imaging (MRI). Journal of Biomechanics, 23, 495-501.

Scott, S.H., \& Winter, D.A. (1990). Internal forces at chronic running injury sites. Medicine and Science in Sports and Exercise, 22, 357-369.

Winter, D.A. (1980). Overall principle of lower limb support during stance phase of gait. Journal of Biomechanics, 13, 923-927.

Zatsiorsky, V., \& Seluyanov, V. (1985). Estimation of the mass and inertia characteristics of the human body by means of the best predictive regression equations. In D.A. Winter, R.W. Norman, R.P. Wells, K.C. Hayes, \& A.E. Patla (Eds.), Biomechanics $I X-B$ (pp. 233-239). Baltimore: University Park Press.

\section{Acknowledgments}

The authors would like to acknowledge the contribution made by Qualisys, Sweden, in providing the movement analysis equipment used in this study. 